\title{
Nivel de implementación de la calidad en sector turístico gallego
}

\author{
José Álvarez García \\ José Antonio Fraiz Brea \\ María de la Cruz Del Río Rama ${ }^{i}$
}

Universidad de Vigo (España)

\begin{abstract}
Resumen: En estas últimas décadas, la aparición de nuevos destinos turísticos con características y precios similares o incluso más bajos, redujeron notablemente la cuota de mercado de España. Por todo ello, las empresas turísticas para enfrentarse a la mayor competencia de los mercados debido en parte al proceso de globalización, han buscado nuevas herramientas que les permitan obtener ventajas competitivas frente a los competidores para poder sobrevivir en un mundo altamente competitivo. Estas estrategias están enfocadas hacia la calidad del turismo y se basaban en la diferenciación del producto ofertado al cliente. La importancia de la calidad nos hace comprender la necesaria investigación, tanto teórica como empírica, que ayude a desarrollar un marco teórico-práctico para las empresas que deseen implantar la calidad como fuente de ventaja competitiva. Por ello en este trabajo nos planteamos dos objetivos básicos, conocer el nivel de implementación de factores críticos de la calidad en las empresas turísticas gallegas que poseen la certificación Q de calidad, y validar la escala utilizada para analizar el nivel de implementación de los mismos.
\end{abstract}

Palabras clave: Turismo; Gestión de la calidad; "Q de Calidad Turística”.

Title: Level of implementation of quality in tourism sector gallego

\begin{abstract}
In recent decades, the emergence of new destinations and price characteristics similar or even lower, significantly reduced the market share of Spain. Therefore, tourism businesses to face increased competition from markets due in part to the globalization process, have sought new tools to gain competitive advantage over competitors to survive in a highly competitive world. These strategies are focused on the quality of tourism and based on product differentiation offered to the customer. The importance of quality makes us understand the necessary research, both theoretical and empirical, to help develop a theoretical and practical framework for companies wishing to implement quality as a source of competitive advantage. Therefore, in this work we considered two basic objectives, knowing the level of implementation of critical factors of quality in tourism enterprises that are certified Galician quality "Q", and validate the scale used to analyze the level of their implementation.
\end{abstract}

Keywords: Tourism; Quality management; “Q for Quality”.

i Facultade de Ciencias Empresariais e Turismo (Campus Ourense). Universidad de Vigo. E-mail1: pepealvarez@uvigo.es; Email2: jafraiz@uvigo.es; E-mail3: delrio@uvigo.es 


\section{Introducción y objetivos}

Varias son las características que definen la economía actual; la internacionalización o globalización y la creciente competición global, todo ello unido a un incremento de la sofisticación y demanda de los clientes, constituyen un reto hoy en día para las empresas del sector turístico.

Por otro lado, la aparición de nuevos destinos turísticos con características y precios similares o incluso más bajos, redujeron notablemente la cuota de mercado de España, lo que ha llevado a que las empresas turísticas deban enfrentarse a la mayor competencia de los mercados debido en parte al proceso de globalización. En este contexto las empresas han buscado nuevas herramientas/ estrategias que les permitan obtener ventajas competitivas frente a los competidores para poder sobrevivir en un mundo altamente competitivo. Estas estrategias estaban enfocadas hacia la calidad del turismo y se basaban en la diferenciación del producto ofertado al cliente.

Así, en la actualidad, "La Calidad" adquiere mayor importancia y protagonismo, que unido a la innovación, podemos afirmar, que son los dos pilares básicos en los que se apoya la competitividad empresarial.

En la demanda turística se han producido cambios importantes, los consumidores cada vez tienen un nivel de exigencia superior unido a la aparición de nuevos destinos competidores, propició que a mediados de los años 90 el sector turístico español decidiera llevar a cabo un cambio estratégico y poner en marcha iniciativas que permitiesen a las empresas avanzar en la mejora de la calidad de sus servicios y productos como símbolo diferenciador en los mercados nacionales e internacionales y como factor competitivo. Como consecuencia, el sector turístico precisa buscar un equilibrio entre cuatro elementos fundamentales: el precio, la calidad y diferenciación de la oferta, la rentabilidad y la sostenibilidad.

Por este motivo la Administración Turística del Estado decide apostar por el concepto de "Calidad Turistica Española”. Esta apuesta por la calidad se plasma en los programas puestos en marcha por la Secretaria General de Turismo como son el Plan de Calidad del Turismo Español (PICTE 2000-2006) y el Plan Horizonte 2020.

En la misma línea, en el año 1996 la Administración Turística del Estado promovió el macroproyecto denominado "Sistema de Calidad Turística Española", que ha permitido desarrollar sistemas de calidad específicos para diversos subsectores turísticos, creando la " $Q$ de Calidad Turística Española" bajo la cual se amparan un conjunto de productos turísticos que cumplen con unos niveles de calidad exigidos y recogidos en las "Normas de Calidad de Servicios", que aseguran estar trabajando en la mejora continua de los mismos con el fin de complacer en todo momento las exigencias de los clientes. El objetivo primordial de la "Q" es el reconocimiento tanto del consumidor nacional como de los consumidores y operadores de los mercados emisores.

Por todo lo comentado hasta este momento, se aprecia que la calidad se ha convertido hoy en día en una de las variables clave de la competitividad que las organizaciones del sector turístico deben incorporar a su estrategia corporativa.

La importancia de la calidad nos hace comprender la necesaria investigación, tanto teórica como empírica, que ayude a desarrollar un marco teórico-práctico para las empresas que deseen implantar la calidad como fuente de ventaja competitiva. Así la calidad y los principios/factores críticos necesarios para su implantación se han convertido en objeto de estudio de numerosos investigadores.

En este trabajo nos planteamos dos objetivos básicos, conocer el nivel de implementación de factores críticos de la calidad en las empresas turísticas que poseen la certificación "Q de Calidad Turística" en la Comunidad Autónoma Gallega y validar la escala utilizada para analizar el nivel de implementación de los factores críticos en el sector turístico.

La novedad del estudio radica en tres razones: (1) se lleva a cabo en empresas del sector turístico, sector donde no existen estudios que analicen la implantación de Sistemas de Gestión de Calidad y sus consecuencias en el funcionamiento de la empresa; (2) las empresas analizadas poseen un Sistema de Gestión de la Calidad basado en normas UNE (182001:2008-hoteles y apartamentos turísticos), denominado Marca Q de Calidad Turística, marca propia del sector y única en el mundo, basada en una normativa que se sitúa en un nivel intermedio entre la ISO 9000 (aseguramiento de la calidad) y el Modelo EFQM (Calidad Total), por lo que la implantación de la misma es compatible con ambas certificaciones.; (3) el estudio se realizará en empresas que posee un Sistema de Gestión de la Calidad intermedio entre el aseguramiento (ISO 9001) y Calidad Total (EFQM), los estudios de Gestión de la Calidad hasta estos momentos se realizaron mayoritariamente en el ámbito del aseguramiento, en base a la norma ISO 9000 ó en Gestión de la Calidad Total, Modelo Europeo de Excelencia (EFQM)

Para poder dar respuesta a estos objetivos el trabajo se estructura en varios apartados, en primer lugar, definimos el marco teórico derivado de la revisión bibliográfica efectuada, abordando el estudio de todos aquellos factores críticos que se consideran como clave para el éxito de la implantación de la Calidad Total. El segundo apartado se dedica a la metodología de trabajo, en el mismo se delimita el ámbito de estudio en nuestro caso las empresas de alojamiento del sector turístico que tengan implantada la "Q de Calidad", el diseño del cuestionario, el proceso de obtención de datos, y las técnicas utilizadas en el análisis de datos. El tercer apartado recoge el análisis de los resultados y en el último se presentan las principales conclusiones e implicaciones de trabajo.

\section{Marco teórico}

En la literatura sobre el tema ha quedado demostrado que para que la implantación de la Gestión de la Calidad Total tenga éxito es necesario tener en cuenta, los principios de la calidad junto con las prácticas, herramientas y 
técnicas que nos permiten aplicarlos (Sitkin et al., 1994; Easton and Jarrell, 1998; Claver et al., 1999; Wilkinson et al., 1998; Zhang, 2000).

Estos factores críticos a tener en cuenta han sido identificados desde tres áreas diferentes: (1) las aportaciones de los gurús de la calidad (Crosby, 1979; Deming, 1982, 1986; Isikawa, 1985; Juran, 1988; Feigenbaum, 1991), (2) modelos para la implantación de la calidad que han surgido (EFQM, Deming, MBNQA, Iberoamericano), y (3) las aportaciones fundamentales de las investigaciones empíricas llevadas a cabo por estudiosos del tema de Gestión de la Calidad Total.

El punto de partida de la GCT han sido las ideas aportadas por los gurús de la calidad (Deming 1982, 1986; Juran 1988; Juran y Gryna, 1993; Ishikawa 1976, 1985; Crosby 1979; Feigenbaum 1991) ya que muestran tanto los puntos fuertes como débiles de la gestión de la calidad, aunque no ofrecen soluciones a todos los problemas encontrados por la empresas en la aplicación de la GCT (Dale, 1999), sí han sido fundamentales para el desarrollo de los estudios posteriores que han identificado los factores críticos de la GCT, en un intento de aportar a las empresas los componentes de la calidad en los que deben centrarse. Kanji (1998) determina que los principios, o factores críticos de éxito, son las áreas clave de la organización que, de gestionarse de forma adecuada, garantizan la mejora de la competitividad y la excelencia empresarial.

Los factores críticos identificados por los investigadores varían de unos a otros, puesto que cada investigador señala los que bajo su punto de vista son los fundamentales para gestionar correctamente la organización, mejorar la competitividad y la excelencia empresarial (Kanji, $1998 b$ ), pero se hace necesaria una sistematización, es decir, llegar a un consenso de cuáles son esos principios de gestión de la calidad.

En este sentido, la primera investigación llevada a cabo para definir o determinar cuáles son los factores críticos o principios básicos de la calidad fue el estudio de Saraph et al. (1989). Su objetivo fue desarrollar y validar empíricamente un instrumento para medir las prácticas de calidad y en el mismo aporta ocho factores críticos recogidos de la literatura publicada por los gurús de la calidad. La línea de investigación que Saraph et al. (1989) inició, es decir, elaborar un instrumento válido para medir las prácticas de gestión de la calidad en las empresas, fue seguida por estudios realizados por Flynn et al. (1994), Ahire et al. (1996) y Rao et al. (1999).

Son muchos los autores que realizaron investigaciones en este sentido, y recogemos sólo algunas de las más importantes en la tabla 1 .

Además de toda la investigación analizada también surgió en un intento de ayudar a las empresas en su implantación de la GCT, los modelos de calidad, que son guías para la implantación o para realizar autoevaluaciones de sus prácticas de calidad en un intento de llevar a cabo la mejora continua en la empresa. Podemos citar como principales modelos el Malcolm Baldrige Nacional Quality Award (EEUU), el modelo de La Fundación Eu- ropea para la Calidad (EFQM), y modelo Premio Deming en Japón.

Todos ellos han agrupado los factores críticos de la Gestión de la Calidad y aunque existen diferencias entre ellos, presentan una serie de elementos comunes (Ritchie y Dale, 2000). El énfasis de estos modelos es el logro de la satisfacción del cliente a través de la mejora continua (tabla 2).

\section{Metodología de investigación}

La metodología empleada en el estudio empírico para conocer el nivel de implementación de factores críticos de la calidad en las empresas de alojamiento turístico que poseen la certificación "Q de Calidad Turística" y validar la escala utilizando el análisis factorial exploratorio.

En primer lugar, se procedió a realizar una base de datos con todos los alojamientos turísticos gallegos con la Marca "Q de Calidad Turística" a partir de la información obtenida de la página Web del ICTE (http://www.icte. es), así nuestra población objeto de estudio queda constituida por 75 alojamientos turísticos (fecha elaboración abril 2010). A continuación se elabora el cuestionario y se envía por correo electrónico acompañado de una carta de presentación de la investigación a realizar a cada una de las entidades que configuran la población objetivo.

En lo referente al cuestionario éste está compuesto de dos partes. Una primera parte, formada por preguntas introductorias con la finalidad de obtener el perfil de la empresa, y su relación con la Q de calidad. Y una segunda que se estructura en una serie de preguntas referidas a la Gestión de la Calidad Total según el modelo EFQM de Excelencia, agrupadas en cinco bloques (variables) que se corresponden con cinco de los nueve criterios del modelo europeo; (1) liderazgo, 5 ítems, (2) Política y estrategia, 3 ítems, (3) Personas, 5 ítems, (4) Alianzas y recursos, 4 ítems, y (5) Procesos, 4 ítems. En la elaboración de los ítems se ha tomado como referencia las preguntas del modelo EFQM1.

Mediante estos ítems se trata de medir el grado de implantación de la gestión de la calidad total en la comunidad gallega. La escala de valoración utilizada para medir los ítems es una escala tipo Likert de 5 puntos utilizada habitualmente en este tipo de estudios (tabla 4).

El proceso de recogida de datos tuvo lugar durante el mes de mayo del presente año, realizándose el mismo a través de un contacto por E-mail con cada una de los alojamientos turísticos seleccionados. En la tabla 5, se recoge la ficha técnica del trabajo de campo.

En la tabla 6, recogemos las fases seguidas en esta investigación empírica.

Por último, en la tabla 7 se describen algunas de las características básicas que constituyen el perfil de las empresas que forman parte de la muestra, siendo las variables utilizadas para su obtención; la distribución geográfica, el tamaño o dimensión de las empresas, y el tipo de establecimiento. 


\begin{tabular}{|c|c|c|}
\hline Autor & Propuesta & Fuente de los factores- Resultados \\
\hline $\begin{array}{l}\text { Saraph et al. } \\
\text { (1989) }\end{array}$ & $\begin{array}{l}\text { Desarrollo de un instrumento de medida de } \\
\text { los factores críticos de la gestión de la calidad, } \\
\text { en Minneapolis EE.UU. }\end{array}$ & $\begin{array}{l}\text { De los conceptos y principios de calidad de los gurús, } \\
\text { incluyendo Deming, Juran, Crosby e Ishikawa. } \\
\text { Identificaron } 8 \text { factores críticos (66 ítems). }\end{array}$ \\
\hline $\begin{array}{l}\text { Flynn et al., } \\
\text { (1994) }\end{array}$ & $\begin{array}{l}\text { Desarrollo de un instrumento de medida de } \\
\text { los factores críticos de la gestión de la calidad. }\end{array}$ & $\begin{array}{l}\text { Literatura empírica y profesional e informes sobre el } \\
\text { uso real de las prácticas en EE.UU. y Japón. } \\
\text { Identificaron } 7 \text { factores críticos ( } 48 \text { ítems). }\end{array}$ \\
\hline Powell (1995) & $\begin{array}{l}\text { Instrumento de medida de los factores críticos } \\
\text { de la calidad }\end{array}$ & $\begin{array}{l}\text { De la literatura de gestión de la calidad. } \\
\text { Identificaron } 12 \text { factores críticos. }\end{array}$ \\
\hline $\begin{array}{l}\text { Anderson et al. } \\
\text { (1995) }\end{array}$ & Análisis de los factores críticos de GCT, & $\begin{array}{l}\text { De los trabajos de Deming. } \\
\text { Identificaron } 7 \text { factores críticos. }\end{array}$ \\
\hline Badri et al. (1995) & $\begin{array}{l}\text { Instrumento adicional al propuesto por } \\
\text { Saraph et al. (1989). Emiratos Árabes }\end{array}$ & $\begin{array}{l}\text { Replica del trabajo de Saraph et al. (1989). } \\
\text { Identificaron } 8 \text { factores críticos (66 ítems). }\end{array}$ \\
\hline $\begin{array}{l}\text { Black y Porter } \\
\text { (1996) }\end{array}$ & $\begin{array}{l}\text { Identificaron un conjunto de factores críticos y } \\
\text { con ellos elaboraron un instrumento para } \\
\text { medir el alcance de la GCT en una } \\
\text { organización. Utilizaron miembros del EFQM. }\end{array}$ & $\begin{array}{l}\text { Malcolm Baldrige National Award Quality model } \\
\text { (MBNAQ). } \\
\text { Identificaron } 10 \text { factores críticos ( } 32 \text { ítems). }\end{array}$ \\
\hline Ahire et al. (1996) & $\begin{array}{l}\text { Desarrollaron escalas para medir los } \\
\text { constructos que identificaron. }\end{array}$ & $\begin{array}{l}\text { Literatura sobre gestión de la calidad, } \\
\text { comportamiento organizacional y gestión de las } \\
\text { operaciones. Literatura conceptual y prácticas de las } \\
\text { organizaciones evidenciadas a través de varios casos } \\
\text { de estudio e investigaciones empíricas. } \\
\text { Identificaron } 12 \text { factores críticos ( } 50 \text { ítems). }\end{array}$ \\
\hline $\begin{array}{c}\mathbf{L i} \\
(1997)\end{array}$ & $\begin{array}{l}\text { Identificar factores críticos en Hospitales en } \\
\text { EE.UU. (sector servicios). }\end{array}$ & $\begin{array}{l}\text { Malcolm Baldrige National Award Quality model } \\
\text { (MBNAQ). } \\
\text { Identificaron } 6 \text { factores críticos. }\end{array}$ \\
\hline $\begin{array}{l}\text { Leal } \\
(1997)\end{array}$ & $\begin{array}{l}\text { Identificaron los factores críticos en un } \\
\text { estudio empírico realizado en } 113 \text { empresa } \\
\text { españolas. }\end{array}$ & $\begin{array}{l}\text { A partir de las aportaciones de Waldman (1994) y } \\
\text { Powell (1995). } \\
\text { Identificaron } 10 \text { principios clave }\end{array}$ \\
\hline $\begin{array}{l}\text { Forza y Fillipine } \\
\qquad(1998)\end{array}$ & Análisis de los factores críticos de GCT & $\begin{array}{l}\text { Combinación del premio Deming, Baldrige y el premio } \\
\text { Europeo de la calidad. } \\
\text { Identificaron } 6 \text { factores críticos. }\end{array}$ \\
\hline $\begin{array}{c}\text { Grandzoly } \\
\text { Gershon (1998) }\end{array}$ & $\begin{array}{l}\text { Desarrollaron y testaron un instrumento para } \\
\text { usar en las investigaciones de GCT. }\end{array}$ & $\begin{array}{l}\text { Utilizaron los } 7 \text { factores críticos propuestos por } \\
\text { Anderson et al. (1994)- variables exógenas y } 7 \text { de } \\
\text { resultados- variable endógenas (productos o } \\
\text { servicios, resultados financieros y operativos, } \\
\text { responsabilidad social, la satisfacción del cliente, la } \\
\text { satisfacción de los empleados, calidad total). } \\
\text { Identificaron } 7 \text { factores exógenos con } 39 \text { ítems y } 6 \\
\text { endógenos con } 23 \text { items. }\end{array}$ \\
\hline $\begin{array}{l}\text { Tamimi } \\
\text { (1998) }\end{array}$ & $\begin{array}{l}\text { Análisis de los factores críticos de GCT, análisis } \\
\text { en Pensilvania }\end{array}$ & $\begin{array}{l}\text { Los } 14 \text { puntos de Deming. } \\
\text { Identificaron } 8 \text { factores ( } 32 \text { ítems). }\end{array}$ \\
\hline Quazi et al. (1998) & $\begin{array}{l}\text { Corroboraron los resultados de un estudio } \\
\text { desarrollado por Saraph et al., 1994, y se } \\
\text { compara con un estudio de Calingo et al. } \\
\text { (1995). }\end{array}$ & $\begin{array}{l}\text { Saraph et al., } 1994 . \\
\text { Identificaron } 16 \text { factores críticos ( } 78 \text { ítems). }\end{array}$ \\
\hline $\begin{array}{l}\text { Thiagarajan y } \\
\text { Zairi } \\
\text { (1998) }\end{array}$ & $\begin{array}{l}\text { Identificaron los factores críticos de la GCT en } \\
\text { Malasia. }\end{array}$ & $\begin{array}{l}\text { De la literatura de críticos y los ordenaron por nivel de } \\
\text { implementación.GCT. } \\
\text { Identificaron } 22 \text { factores. }\end{array}$ \\
\hline $\begin{array}{l}\text { Easton y Jarrel } \\
\text { (1998) }\end{array}$ & $\begin{array}{l}\text { Identificaron los factores críticos en } 108 \\
\text { empresas en EE.UU. }\end{array}$ & $\begin{array}{l}\text { Análisis de la literatura y Malcolm Baldrige National } \\
\text { Award Quality Model. } \\
\text { Identificaron } 9 \text { factores críticos. }\end{array}$ \\
\hline $\begin{array}{l}\text { Joseph et al. } \\
\text { (1999) }\end{array}$ & $\begin{array}{l}\text { Identificaron los factores críticos de la GCT en } \\
\text { la India. }\end{array}$ & $\begin{array}{l}\text { Saraph et al., } 1994 . \\
\text { Identificaron } 10 \text { factores (106 ítems). }\end{array}$ \\
\hline Rao et al. (1999) & $\begin{array}{l}\text { Desarrollaron un instrumento válido para } \\
\text { medir las principales dimensiones de la } \\
\text { gestión de la calidad en el contexto } \\
\text { internacional. }\end{array}$ & $\begin{array}{l}\text { Análisis de la literatura y Malcolm Baldrige National } \\
\text { Award Quality Model. } \\
\text { Identificaron } 13 \text { factores con } 62 \text { ítems. }\end{array}$ \\
\hline $\begin{array}{l}\text { Zhang et al. } \\
\qquad(2000)\end{array}$ & $\begin{array}{l}\text { Desarrollo un modelo de métodos de gestión } \\
\text { de la calidad basado principalmente en el de } \\
\text { Mann y Kehoe (1994). }\end{array}$ & $\begin{array}{l}\text { Revisión de la literatura. } \\
\text { Proponen } 11 \text { factores críticos y } 83 \text { métodos. }\end{array}$ \\
\hline $\begin{array}{l}\text { Motwani et al. } \\
\text { (2001) }\end{array}$ & $\begin{array}{l}\text { Instrumento adicional al propuesto por } \\
\text { Saraph, Benson y Schroeder. Se muestran las } \\
\text { relaciones entre los factores críticos. }\end{array}$ & $\begin{array}{l}\text { De la literatura de gestión de la calidad. } \\
\text { Identificaron } 7 \text { factores críticos ( } 45 \text { ítems). }\end{array}$ \\
\hline $\begin{array}{l}\text { Sureshchandar et } \\
\text { al., } \\
\text { (2001) }\end{array}$ & $\begin{array}{l}\text { Identificaron un conjunto de factores críticos } \\
\text { de gestión de la calidad en el sector servicios }\end{array}$ & $\begin{array}{l}\text { Revisión de la literatura existente. } \\
\text { Identificaron } 12 \text { factores. }\end{array}$ \\
\hline $\begin{array}{l}\text { Behara } \\
(2001)\end{array}$ & $\begin{array}{l}\text { Identificaron un conjunto de factores críticos } \\
\text { de gestión de la calidad en el sector servicios. }\end{array}$ & $\begin{array}{l}\text { Malcolm Baldrige National Award Quality model } \\
\text { (MBNAQ). } \\
\text { Identificarón } 11 \text { factores críticos ( } 69 \text { items). }\end{array}$ \\
\hline $\begin{array}{c}\text { Santos y } \\
\text { Escanciano (2002) } \\
\end{array}$ & $\begin{array}{l}\text { Identificaron un conjunto de factores críticos } \\
\text { de gestión de la calidad }\end{array}$ & Identificaron 5 factores críticos. \\
\hline $\begin{array}{l}\text { Anthony et al. } \\
\quad(2002)\end{array}$ & $\begin{array}{l}\text { Identificar los factores de éxito de la GCT en } \\
\text { empresas de Hong Kong. }\end{array}$ & $\begin{array}{l}\text { Saraph et al., } 1994 . \\
\text { Identificaron } 7 \text { factores críticos de gestión de la } \\
\text { calidad ( } 38 \text { ítems) en industrias de Hong Kong. }\end{array}$ \\
\hline $\begin{array}{l}\text { Conca et al. } \\
\text { (2004) }\end{array}$ & $\begin{array}{l}\text { Identificar los factores críticos de gestión de la } \\
\text { calidad. }\end{array}$ & $\begin{array}{l}\text { Identificaron } 8 \text { factores críticos de gestión de la } \\
\text { calidad. }\end{array}$ \\
\hline
\end{tabular}

Tabla 1: Factores críticos en la literatura. Fuente: Elaboración propia

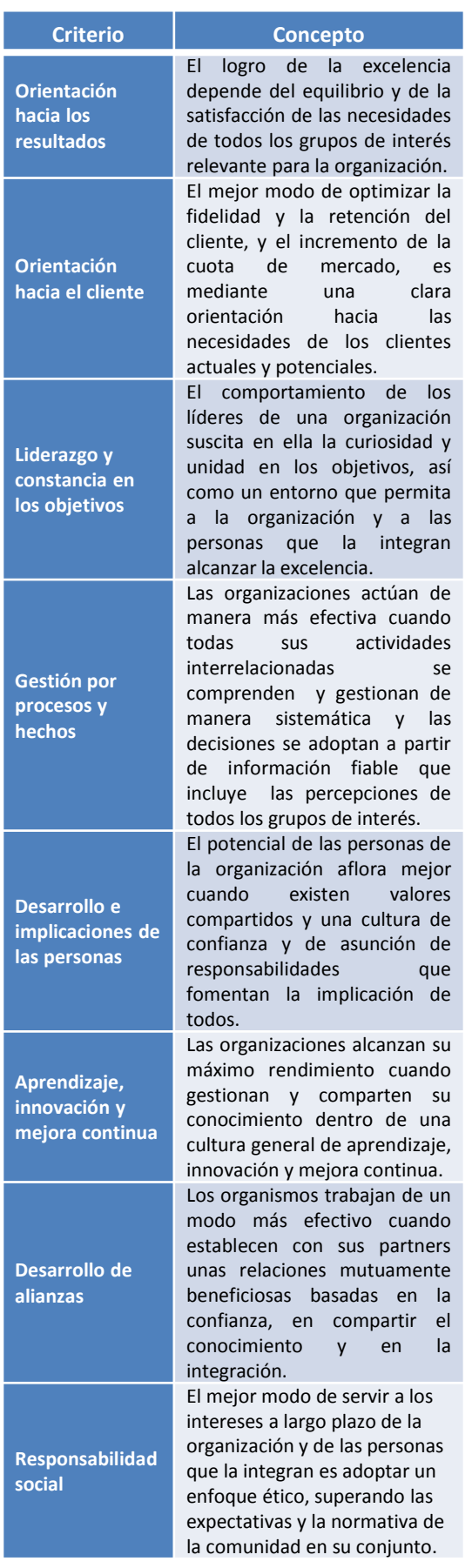

Tabla 2: Conceptos fundamentales del modelo efqm de excelencia. Fuente: EFQM (1999) 


\begin{tabular}{|c|c|}
\hline LID1 & Existe compromiso de la dirección en el programa de calidad \\
\hline LID2 & $\begin{array}{l}\text { Se implica activamente la dirección en las actividades de mejora, se anima al personal a emprender } \\
\text { mejoras en el día a día }\end{array}$ \\
\hline LID3 & $\begin{array}{l}\text { Se reúne habitualmente la dirección con clientes, proveedores y otros agentes externos y emprende con } \\
\text { ellos acciones de mejora }\end{array}$ \\
\hline LID4 & Existe un claro clima de diálogo entre empresa y empleados \\
\hline LID5 & La dirección reconoce los esfuerzos y las mejoras alcanzadas por el personal, proveedores, etc. \\
\hline PO2 & $\begin{array}{l}\text { Estas estrategias y planes se despliegan en objetivos realistas y son comunicados a los empleados para que } \\
\text { los conozcan y cumplan }\end{array}$ \\
\hline PO3 & $\begin{array}{l}\text { Se revisa periódicamente la estrategia, los planes de negocio y los objetivos para detectar desviaciones y, } \\
\text { si procede, modificarlos }\end{array}$ \\
\hline PE3 & Se desarrollan planes de formación específicos. \\
\hline PE4 & $\begin{array}{l}\text { Se fomenta que las personas asuman responsabilidades y se impliquen en actividades de mejora, trabajen } \\
\text { en equipo, etc. }\end{array}$ \\
\hline PE5 & Los empleados consideran que están bien informados y que sus opiniones se valoran. \\
\hline AR1 & Se establecen acuerdos a largo plazo con los principales proveedores \\
\hline AR2 & $\begin{array}{l}\text { Se utilizan adecuadamente los recursos económicos y financieros de forma que se garantice el éxito de los } \\
\text { planes de negocio. }\end{array}$ \\
\hline AR3 & Se recoge y gestiona toda la información importante, y esta es utilizada por el personal correspondiente. \\
\hline AR4 & En general, se realiza una gestión de alianzas y recursos acorde con los planes de negocio. \\
\hline PRO1 & $\begin{array}{l}\text { Se establece un sistema de gestión de procesos, especialmente los procesos claves, en línea con los planes } \\
\text { de negocio }\end{array}$ \\
\hline
\end{tabular}

Tabla 3: Ítems de cuestionario

\begin{tabular}{|c|c|}
\hline $\begin{array}{c}\text { Nivel de } \\
\text { implantación }\end{array}$ & Orientación \\
\hline 1 & $\begin{array}{l}\text { No, o prácticamente } \\
\text { no implantado }(0 \%)\end{array}$ \\
\hline 2 & $\begin{array}{l}\text { Sí, en algunos casos o } \\
\text { en ocasiones muy } \\
\text { puntuales }(25 \%)\end{array}$ \\
\hline 3 & $\begin{array}{l}\text { Sí, en la mayoría de } \\
\text { los casos }(50 \%)\end{array}$ \\
\hline 4 & $\begin{array}{l}\text { Sí, casi siempre y de } \\
\text { forma más o menos } \\
\text { sistemática ( } 75 \%)\end{array}$ \\
\hline 5 & $\begin{array}{l}\text { Sí, siempre y de forma } \\
\text { sistemática }(100 \%)\end{array}$ \\
\hline
\end{tabular}

Tabla 4: Escala de medición

Universo de población

Ámbito Geográfico

Población

Tamaño de la muestra

Índice de respuesta

Error muestral

Nivel de confianza

Método de recogida de información

Fecha del trabajo de campo

Tabla 5: Ficha técnica trabajo de campo. Fuente: Elaboración propia

Etapa 1: Elaboración del

cuestionario

Etapa 2: Recogida de datos Etapa 3: Tabulación de los datos

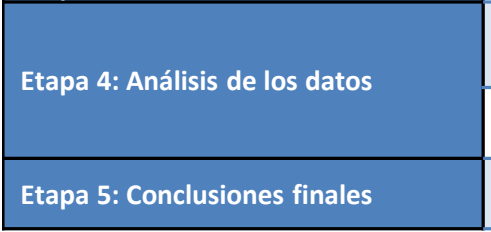

Tabla 6: Fases de la investigación empírica. Fuente: Elaboración propia Programa Estadístico SPSS

los resultados obtenidos

\section{Ficha Técnica}

Alojamientos hoteleros con " $Q$ de Calidad"

Galicia

75 alojamientos hoteleros

39 establecimientos

$52 \%$

$10,9 \%$

$95 \%(Z=1,96 ; p=q=0,5)$

E-mail y teléfono

Mayo 2010

Nos hemos basado en el cuestionario de Modelo Europeo de la Calidad

La hemos realizado a través de Email

\begin{tabular}{|l|l}
\hline Análisis descriptivo & Primer
\end{tabular} (Paquete Estadístico SPSS) objetivo Análisis Factorial Exploratorio Segundo objetivo

Extraemos las conclusiones finales a partir de 


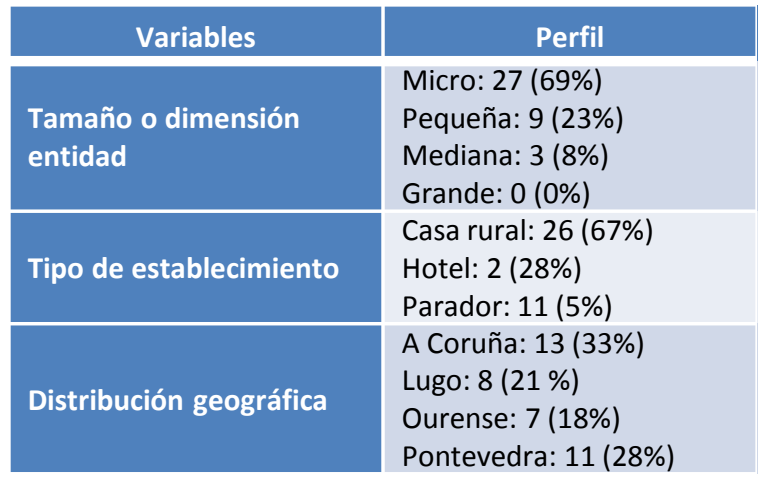

Tabla 7: Perfil muestra. Fuente: Elaboración propia

\section{Análisis e interpretación de los resultados}

\section{Análisis descriptivo}

Las fases y técnicas estadísticas utilizadas en este análisis se detallan en la tabla 8.

\begin{tabular}{|l|l|}
\multicolumn{1}{|c|}{ FASE ANÁLISIS } & \multicolumn{1}{c|}{ TÉCNICA ESTADÍSTICA } \\
\hline Análisis descriptivo & Estadístico descriptivo (SPSS) \\
\hline Fiabilidad y validez escalas & Análisis factorial exploratorio (SPSS) \\
\hline
\end{tabular}

Tabla 8: Fases y técnicas de análisis

En la tabla 9 aparecen recogidos los datos globales de los criterios analizados, donde se observa que los puntos fuertes o criterios más valorados por parte de las entidades encuestadas son el Liderazgo (84,71\%), junto con la Política y Estrategia (83,41\%), seguidos muy de cerca del criterio Personas (78,66\%), Alianzas y Recursos $(78,20 \%)$ y Procesos (77,82\%). Por lo que queda demostrado que el nivel de implantación de los factores críticos es muy elevado al presentar todos ellos niveles de implantación superiores al $77 \%$. de sus altas puntuaciones se podrían mejorar serían el que los líderes de la organización lleven a cabo una mayor interacción con clientes, proveedores y otros agentes externos con objeto de establecer alianzas y/o acciones de mejora conjuntas. Por otra parte, los líderes deben de reforzar la cultura de excelencia dentro de la empresa, implicándose directamente y reconociendo de forma transparente los esfuerzos y mejoras alcanzadas por el personal, clientes, proveedores y otros colaboradores.

La valoración media global obtenida en el criterio de Política y Estrategia ha sido de un 4,17, la cual resulta muy próxima a la del Liderazgo. De los resultados alcanzados se desprende la existencia de planes estratégicos y/o planes de negocio en línea con la visión y valores de la empresa, los cuales se despliegan en objetivos realistas que son comunicados internamente para su conocimiento a todos los miembros de la organización.

El criterio Personas ha sido valorado de forma global con un 3,93 , lo que le sitúa en tercer lugar en cuanto a valoración. De las puntuaciones individuales obtenidas en cada una de las cuestiones formuladas para el criterio de las personas cabe destacar de modo general la adecuada gestión de los recursos humanos en línea con la estrategia y planes de negocio de la empresa y que los empleados están bien informados y sus opiniones se valoran. También se fomenta y apoya que las personas asuman responsabilidades y se impliquen en actividades de mejora.

Como aspectos menos valorados, y por lo tanto a mejorar sería el que la dirección de la empresa desarrollase planes de formación específicos para su personal y que introdujese mejoras en la gestión de las personas a partir de los datos obtenidos de las encuestas de satisfacción del personal o de reuniones periódicas con los empleados.

En cuanto al criterio Alianzas y Recursos, la valoración global obtenida es de 3,91, ligeramente inferior a los criterios anteriores. Como factores más positivos se encuentran la recogida y gestión de toda la información im-

\begin{tabular}{|l|c|c|c|c|c|c|}
\hline & Rango & Mínimo & Máximo & Media aritmética & Desv. típ. & Varianza \\
\hline Liderazgo (LID) & 3,40 & 1,60 & 5,00 & $4,2359(84,71 \%)$ & 0,83964 & 0,705 \\
\hline Política y estrategia (PO) & 4,00 & 1,00 & 5,00 & $4,1709(83,41 \%)$ & 0,92044 & 0,847 \\
\hline Personas (PE) & 3,60 & 1,20 & 4,80 & $3,9333(78,66 \%)$ & 0,97800 & 0,956 \\
\hline Alianzas y recursos (AR) & 4,25 & 0,75 & 5,00 & $3,9103(78,20 \%)$ & 0,91135 & 0,831 \\
\hline Procesos (PRO) & 3,25 & 1,75 & 5,00 & $3,8910(77,82 \%)$ & 0,72274 & 0,522 \\
\hline
\end{tabular}

Tabla 9: Estadísticos descriptivos

A continuación se procede a un análisis más pormenorizado de cada uno de los cinco criterios y sus respectivas cuestiones/ítems con los que los hemos medido (gráfico 1).

Como ya se ha mencionado, el criterio Liderazgo es el más valorado con una media aritmética global de 4,23. Los aspectos más positivos a destacar dentro de esta categoría son los correspondientes al compromiso de la Dirección en el programa de calidad y a que ésta se implica activamente en las actividades de mejora y se anima al personal a emprender mejoras día a día.

Algunas cuestiones relativas al Liderazgo que a pesar portante y su utilización por el personal correspondiente y la eficiente gestión de los recursos económicos y financieros para garantizar el éxito de los planes de negocio.

Una posible mejora de esta categoría sería la referente a la gestión de las alianzas externas, ya que, los resultados obtenidos indican que habría que establecer más acuerdos estratégicos a largo plazo con los principales proveedores con objeto de buscar cooperaciones que permitan crear valor y maximizarlo. Por otra parte, las empresas señalan de forma general que también habría que mejorar la gestión de las alianzas y recursos para que ésta 

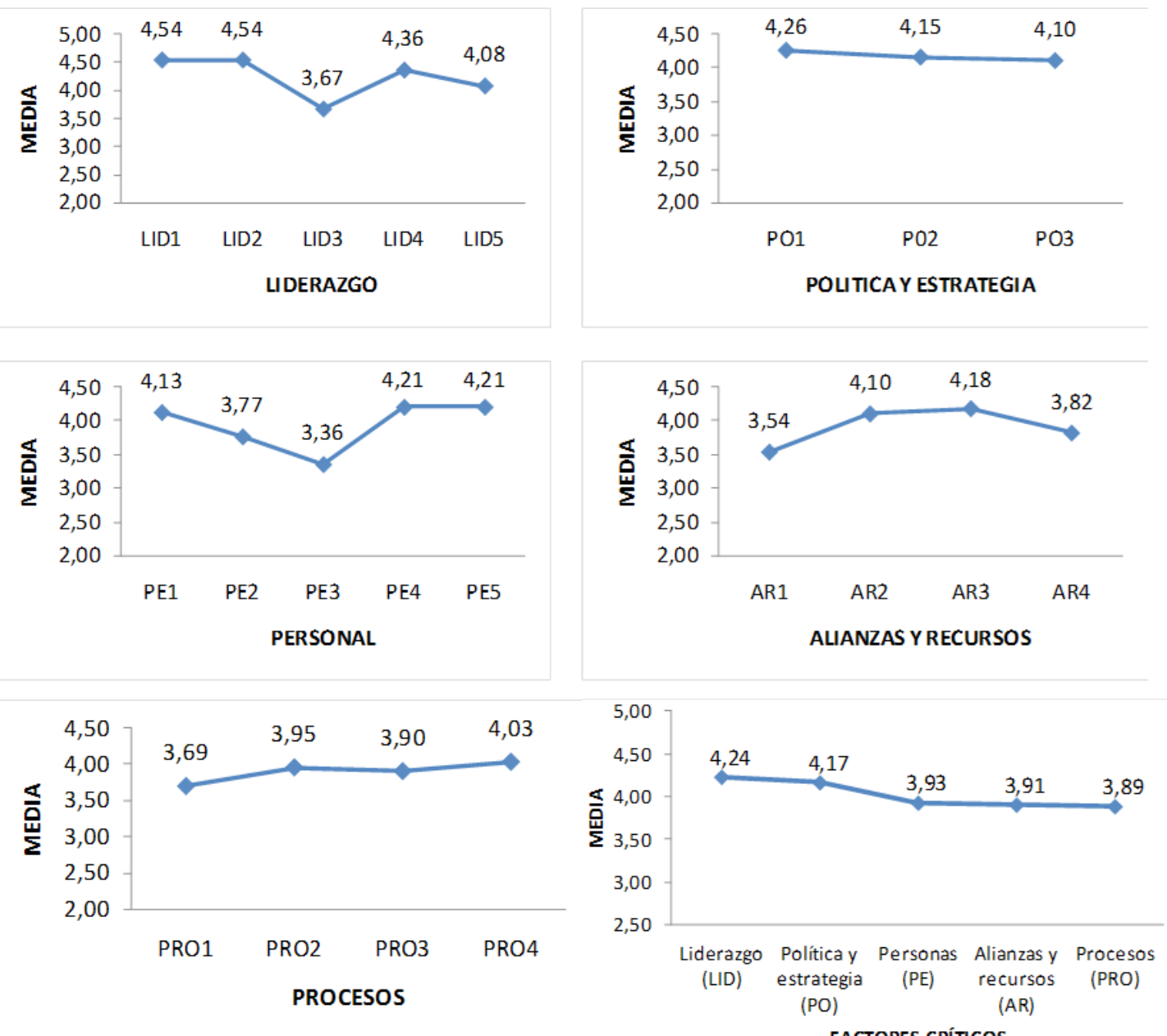

FACTORES CRÍTICOS

Gráfico 1: Factores críticos de la calidad

fuese más acorde con la estrategia y los planes de negocio de la empresa.

El último criterio analizado es el referente a los Procesos que fue valorado de forma global con 3,89. Como puntos fuertes se encuentran la introducción de mejoras en los servicios como consecuencia de la realización de encuestas de satisfacción, reuniones con los principales clientes, gestión de quejas y reclamaciones, y la inserción de mejoras en los procesos como consecuencia de los resultados de sus indicadores.

Dentro de los aspectos más débiles y que habría que mejorar en este criterio se encuentra el sistema de gestión de los procesos, especialmente los procesos claves, para facilitar el plan estratégico de la empresa. Sin dejar de lado la realización de estudios de mercado para conocer las necesidades y expectativas de los clientes y poder diseñar nuevos productos y servicios o introducir mejoras en los existentes.

Con este análisis hemos cumplido con nuestro primer objetivo: conocer el nivel de implementación de factores críticos de la calidad en las empresas turísticas que poseen la certificación $\mathrm{Q}$ de calidad.

\section{Validación de la escala}

\section{Fiabilidad}

Para cumplir con nuestro segundo objetivo, validar la escala utilizada para analizar el nivel de implementación de los factores críticos en el sector turístico, realizamos un análisis de los instrumentos de medida, lo que nos permitirá comprobar si los factores críticos han sido medidos adecuadamente a través de los ítems establecidos en el cuestionario. Es decir, tenemos que comprobar si las escalas utilizadas para medir los factores críticos son una medida fiable y válida de los mismos.

En este sentido, para validar las escalas de medida propuestas hemos seguido las recomendaciones metodológicas de Churchill (1979) y de Anderson y Gerbing (1988), evaluando las propiedades psicométricas de dichas escalas: unidimensionalidad, validez y fiabilidad.

Para el cálculo de la fiabilidad se ha utilizado el método de consistencia interna, por ser el más utilizado según Sánchez y Sarabia (1999). En dicho método se realizan los siguientes análisis para contrastar la fiabilidad de las escalas: (1) Correlación elemento-total de cara a garantizar 
la consistencia interna de las escalas; en este proceso de depuración suprimimos aquellos indicadores que presenten una baja correlación elemento-total, de tal forma que se eliminan aquellos indicadores cuya correlación total no alcance el valor mínimo generalmente aceptado de 0,3; (2) Estimación del a de Cronbach ${ }^{2}$, para cada uno de los elementos a medir y también se calcula el $a$ de Cronbach estandarizado para el total de la muestra. De forma generalizada la literatura utiliza el estadístico $a$ de Cronbach como medida de la fiabilidad y el valor estandarizado recomendado debe ser superior al mínimo exigido de 0,7 (Nunnally, 1979), en nuestro caso la mayoría superan al 0,8 recomendado por determinados autores para estudios confirmatorios (Luque, 2000).

Mediante el análisis de la correlación ítem-total, comprobamos que no existe ningún ítem con una correlación por debajo del mínimo recomendado (Nurossi, 1993) de 0,3. Los estadísticos Alpha de Cronbach, de cada una de las escalas, toma valores superiores al 0,7 recomendado por Nunnally (1978), lo que nos indica la adecuada consistencia interna de las mismas.

\section{Unidimensionalidad (Análisis Factorial Explora- torio)}

Una parte esencial en la especificación de una escala y su validación es la comprobación de su unidimensionalidad. Esta característica supone que, bajo el conjunto de indicadores y elementos que la configuran, subyace un único concepto común que da unión al constructo. Para confirmarla realizamos un Análisis Factorial Exploratorio (de componentes principales con rotación varimax) que permite identificar las dimensiones subyacentes en cada uno de los constructos. Convirtiéndose además en el último paso para corroborar la fiabilidad de las escalas.

Como paso previo a la realización del análisis factorial, es necesario especificar que los datos obtenidos mediante el cuestionario son adecuados para realizar dicho análisis. Para ello es necesario examinar la matriz de correlaciones y comprobar si efectivamente es pertinente continuar con el mismo.

De este modo (tabla 11): (1) comprobamos que en la matriz de correlaciones entre todas las variables disponibles existe un número significativo de correlaciones eleva-

\begin{tabular}{|c|c|c|c|c|c|c|}
\hline \multirow{2}{*}{ Ítem } & \multicolumn{2}{|c|}{$\begin{array}{l}\text { Correlación } \\
\text { Ítem-total }\end{array}$} & \multicolumn{2}{|c|}{$\begin{array}{l}\text { Alfa si se } \\
\text { elimina el } \\
\text { ítem }\end{array}$} & \multirow{2}{*}{$\begin{array}{l}\text { Coeficiente } \\
\alpha \text { de cronbrach }\end{array}$} & \multirow{2}{*}{$\begin{array}{l}\text { Ítems } \\
\text { eliminados }\end{array}$} \\
\hline & Inicial & Final & Inicial & $\begin{array}{l}\mathrm{Fi} \\
\mathrm{n} \\
\mathrm{al}\end{array}$ & & \\
\hline \multicolumn{7}{|c|}{ Análisis de la fiabilidad de la escala liderazgo } \\
\hline LID1 & 0,694 & & 0,893 & & \multirow{5}{*}{$\begin{array}{l}\alpha=0,900 \\
\alpha \\
\text { estandarizado } \\
=0,902\end{array}$} & \multirow{5}{*}{$\begin{array}{l}\text { No se elimina } \\
\text { ningún ítem }\end{array}$} \\
\hline LID2 & 0,731 & & 0,882 & & & \\
\hline LID3 & 0,761 & & 0,875 & & & \\
\hline LID4 & 0,759 & & 0,877 & & & \\
\hline LID5 & 0,845 & & 0,856 & & & \\
\hline \multicolumn{7}{|c|}{ ANÁLISIS DE LA FIABILIDAD DE LA ESCALA POLÍTICA Y ESTRATEGIA } \\
\hline PO1 & 0,837 & & 0,826 & & \multirow{3}{*}{$\begin{array}{l}\alpha=0,899 \\
\alpha \\
\text { estandarizado } \\
=0,902\end{array}$} & \multirow{3}{*}{$\begin{array}{l}\text { No se elimina } \\
\text { ningún ítem }\end{array}$} \\
\hline PO2 & 0,814 & & 0,845 & & & \\
\hline $\mathrm{PO} 3$ & 0,759 & & 0,899 & & & \\
\hline \multicolumn{7}{|c|}{ ANÁLISIS DE LA FIABILIDAD DE LA ESCALA : ALIANZAS Y RECURSOS } \\
\hline AR1 & 0,752 & & 0,887 & & \multirow{4}{*}{$\begin{array}{l}\alpha=0,904 \\
\alpha \\
\text { estandarizado } \\
=0,905\end{array}$} & \multirow{4}{*}{$\begin{array}{l}\text { No se elimina } \\
\text { ningún ítem }\end{array}$} \\
\hline AR2 & 0,756 & & 0,886 & & & \\
\hline AR3 & 0,830 & & 0,859 & & & \\
\hline AR4 & 0,805 & & 0,868 & & & \\
\hline \multicolumn{7}{|c|}{ ANÁLISIS DE LA FIABILIDAD DE LA ESCALA PERSONAS } \\
\hline PE1 & 0,830 & & 0,878 & & \multirow{5}{*}{$\begin{array}{l}\alpha=0,910 \\
\alpha \\
\text { estandarizado } \\
=0,912\end{array}$} & \multirow{5}{*}{$\begin{array}{l}\text { No se elimina } \\
\text { ningún ítem }\end{array}$} \\
\hline PE2 & 0,596 & & 0,926 & & & \\
\hline PE3 & 0,812 & & 0,886 & & & \\
\hline PE4 & 0,823 & & 0,880 & & & \\
\hline PE5 & 0,834 & & 0,877 & & & \\
\hline \multicolumn{7}{|c|}{ ANÁLISIS DE LA FIABILIDAD DE LA ESCALA PROCESOS } \\
\hline PRO1 & 0,658 & & 0,713 & & $\alpha=0,793$ & \multirow{4}{*}{$\begin{array}{l}\text { No se elimina } \\
\text { ningún ítem }\end{array}$} \\
\hline PRO2 & 0,590 & & 0,748 & & & \\
\hline PRO3 & 0,685 & & 0,697 & & estandarizado & \\
\hline PRO4 & 0,495 & & 0,791 & & $=0,791$ & \\
\hline
\end{tabular}

Tabla 10: Análisis de la fiabilidad de las escalas de medida das $(>0,5)$, y además el determinante de la matriz de correlaciones toma un valor próximo a cero en todas las escalas; (2) Test de Esfericidad de Bartlett, en nuestro caso, podemos rechazar en todas la escalas esta hipótesis ya que el valor del test es elevado y está asociado a un nivel de significación inferior al 0,05; (3) Test de adecuación de la medida de Kaiser-Meyer-Olkin (KMO) en nuestro caso presenta valores superiores a 0,7 ; (4) El Índice $M S A$, es inaceptable para valores inferiores a 0,5 ; en ningún caso presenta valores inferiores, la mayoría un valor superior a 0,7 .

La aplicación del análisis factorial no supuso la eliminación de ningún ítem. Como se refleja en la tabla 12, en todos los casos las cargas factoriales son superiores a 0,5 no considerando como significativas cargas factoriales inferiores a 0,3 (Hair et al., 1999) ${ }^{3}$. En todas las escalas el porcentaje acumulado de varianza explicada es superior al $50 \%$. De igual modo el coeficiente de de Cronbach, como un indicador de la fiabilidad de las escalas, supera el valor recomendado de 0,7 . Incluso algunos ítems superan el 0,8, mínimo más exigente propuesto por Grande y Abascal (1999).

Así, a partir de los datos alcanzados en los análisis realizados para corroborar la fiabilidad, podemos concluir que las escalas propuestas resultan altamente fiables, estando por 


\begin{tabular}{|c|c|c|c|c|c|}
\hline & Matriz de correlaciones & $\begin{array}{l}\text { Determinante de la } \\
\text { matriz de correlaciones }\end{array}$ & $\begin{array}{c}\text { Test de esfericidad de } \\
\text { Bartlett }\end{array}$ & $\begin{array}{c}\text { Medida de } \\
\text { adecuación de la } \\
\text { muestra }\end{array}$ & $\begin{array}{l}\text { Índice de } \\
\text { KMO }\end{array}$ \\
\hline Liderazgo & Variables correlacionadas & 0,022 & 135,846 sig 0,000 & $(0,702-0,719)$ & 0,752 \\
\hline $\begin{array}{l}\text { Política y } \\
\text { estrategia }\end{array}$ & Variables correlacionadas & 0,141 & 70,810 sig 0,000 & $(0,695-0,720)$ & 0,738 \\
\hline Personas & Variables correlacionadas & 0,021 & 136,676 sig 0,000 & $(0,855-0,800)$ & 0,845 \\
\hline $\begin{array}{l}\text { Alianzas y } \\
\text { recursos }\end{array}$ & Variables correlacionadas & 0,075 & 92,889 sig 0,000 & $(0,869-0,837)$ & 0,839 \\
\hline Procesos & Variables correlacionadas & 0,263 & 47,809 sig 0,000 & $(0,694-0,711)$ & 0,710 \\
\hline
\end{tabular}

Tabla 11: Indicadores del grado de asociación entre variables

\begin{tabular}{|c|c|c|c|c|c|}
\hline Ítems & $\begin{array}{c}\text { Factores } \\
\text { identificados }\end{array}$ & $\begin{array}{l}\text { Variables que } \\
\text { recoge el factor }\end{array}$ & $\begin{array}{c}\text { Peso de cada } \\
\text { variable en el } \\
\text { factor }\end{array}$ & $\begin{array}{c}\% \text { de } \\
\text { información } \\
\text { explicada }\end{array}$ & $\begin{array}{c}\alpha \text { de } \\
\text { Cronbach }\end{array}$ \\
\hline Liderazgo & F1: Liderazgo & $\begin{array}{l}\text { LID1 } \\
\text { LID2 } \\
\text { LID3 } \\
\text { LID4 } \\
\text { LID5 }\end{array}$ & $\begin{array}{l}0,806 \\
0,843 \\
0,847 \\
0,843 \\
0,901\end{array}$ & $71,99 \%$ & $\alpha=0,900$ \\
\hline $\begin{array}{l}\text { Política y } \\
\text { estrategia }\end{array}$ & $\begin{array}{l}\text { F1: Política y } \\
\text { estrategia }\end{array}$ & $\begin{array}{l}\mathrm{PO} 1 \\
\mathrm{PO} 2 \\
\mathrm{PO} 3\end{array}$ & $\begin{array}{l}0,933 \\
0,889 \\
0,922\end{array}$ & $83,62 \%$ & $\alpha=0,899$ \\
\hline Personas & F1: Personas & $\begin{array}{l}\text { PE1 } \\
\text { PE2 } \\
\text { PE3 } \\
\text { PE4 } \\
\text { PE5 }\end{array}$ & $\begin{array}{l}0,900 \\
0,714 \\
0,886 \\
0,895 \\
0,904\end{array}$ & $74,49 \%$ & $\alpha=0,910$ \\
\hline $\begin{array}{l}\text { Alianzas y } \\
\text { recursos }\end{array}$ & $\begin{array}{l}\text { F1: Alianzas y } \\
\text { recursos }\end{array}$ & $\begin{array}{l}\text { AR1 } \\
\text { AR2 } \\
\text { AR3 } \\
\text { AR4 }\end{array}$ & $\begin{array}{l}0,858 \\
0,864 \\
0,911 \\
0,894\end{array}$ & $77,78 \%$ & $\alpha=0,904$ \\
\hline Procesos & F1: Procesos & $\begin{array}{l}\text { PRO1 } \\
\text { PRO2 } \\
\text { PRO3 } \\
\text { PRO4 }\end{array}$ & $\begin{array}{l}0,827 \\
0,773 \\
0,845 \\
0,689\end{array}$ & $61,74 \%$ & $\alpha=0,793$ \\
\hline
\end{tabular}

Tabla 12: Estudio de la unidimensionalidad

tanto libre de errores aleatorios y capacitadas para proporcionar resultados consistentes, siendo además todas las escalas de medida unidimensionales.

\section{Conclusiones}

La calidad se ha convertido en necesaria para lograr el éxito en un mercado cada vez más competitivo, así hoy en día la calidad de servicio está siendo empleada, cada vez más por multitud de organizaciones como su principal herramienta competitiva, dada la dificultad que implica su imitación por parte de las empresas competidoras. Por otro lado, la certificación "Q" marca un valor añadido frente a la competencia ya que implica un mayor conocimiento de las expectativas de los clientes y tendencias del mercado y proporciona un plus de motivación y profesio- nalización del personal.

De los factores críticos analizados en el estudio, los más valorados han sido el Liderazgo, Política y Estrategia y Personas con resultados superiores al $80 \%$, destacando el compromiso de la Dirección en el programa de calidad, su implicación en las actividades de mejora, animando al personal a emprender mejoras día a día, y por otro lado realiza una adecuada gestión de los recursos humanos en línea con la estrategia y planes de negocio de la empresa.

Los agentes menos valorados han resultado ser Alianzas y Recursos y Procesos por lo que consideramos que habría que mejorar la gestión de las alianzas y recursos para que éstas fuesen más acordes con la estrategia y los planes de negocio de la empresa., así como mejorar el sistema de gestión de los procesos, especialmente los procesos claves, para facilitar el plan estratégico de la 
empresa. Sin dejar de lado la realización de estudios de mercado para conocer las necesidades y expectativas de los clientes.

Como implicaciones prácticas para los directivos en base a las áreas a mejorar planteamos: (1) la necesidad de realizar reuniones periódicas de la dirección con los agentes con los que mantiene relaciones (clientes, proveedores...) con el objetivo de analizar las situaciones desde diferentes puntos de vista y emprender acciones de mejora conjuntas; (2) un aspecto primordial para las empresas a realizar, es la revisión periódica de las estrategias, planes de negocio y los objetivos con la finalidad de detectar las desviaciones y poderlas corregir a tiempo; (3) las empresas deben concienciarse de la necesidad de la formación dentro de la empresa, y poner en marcha planes de formación; (4) las empresas deben tener muy en cuenta la satisfacción de su personal, por lo que tendrá que poner en marcha mecanismos que le permitan medirla dada la importancia de los RRHH en este sector; (5) la necesidad de establecimiento de acuerdos con los proveedores y que estos sean a largo plazo; (6) establecimiento de un sistema de gestión de procesos en línea con los planes de negocio, de forma que se controlen y se realice una mejora continua de los mismos. En esta línea, sería muy importante implantar programas para encontrar las pérdidas de tiempo y costes en todos los procesos y llevar a cabo estudios de mercado que permitan conocer las necesidades actuales y futuras de los clientes, lo que permitiría introducir mejoras en todos los servicios y procesos.

\section{Bibliografía}

Ahire, L.S. y Golhar, D.Y.

1996 "Quality management in large vs small". Journal of Small Business Management, April: 1-13.

Anderson, J.C. y Gerbing, D.W.

1988 "Structural Equation Modeling in Practice: a Review and Recommended Two-step Approach". Psychological Bulletin, 103(3): 411-423.

Anderson, J., Rungtusanatham, M., Schroeder, R.G. y Devaraj, S.

1995 "A Path analytic model of a theory of Quality Management underlying the Deming Management Method: preliminary findings". Decisions Science, 26 (5): 637-657.

Anthony, J., Leung, K.. y Knowless, G.

2002 "Critical Success Factors of TQM Implementation in Hong Kong Industries". International Journal of Quality and Reliability Management, 19: 551-566.

Badri, M.A., Davis, D. y Davis, D.

1995 "A study of measuring the critical factors of quality management"., International Journal of Quality \& Reliability Management, 12 (2): 36-53.

Behara, R.

2001 "Analysis of quality management pratices in services". The current issue and full text archive of this journal is available, 18 (6): 584-601,

Black, S. y Porter, L.J.

1995 "An empirical model for total quality management". Total Quality Management, 6 (2): 149-164.
Churchill, G.A.

1979 "A Paradigm for Developing Better Measures of Marketing Constructs. Journal of Marketing Research, 16, febrero: 64-73.

Claver, E., Llopis, J. y Tarí, J.J.

1999 Calidad y dirección de empresas. Civitas, Madrid.

Conca, F.J., Llopis, J. y Tarí, J.J.

2004 "Development of a measure to assess quality management in certified firms". European Journal of $\mathrm{Op}$ erational Research, 156 (3): 683-697.

Crosby, B.P

1979 Quality is Free (New York, mcgraw-Hill).

Deming, W.E.

1982 Out of the Crisis. Auality, Productivity and Competitive Position. MIT, Cambridge. Edición Española: Calidad, productividad y competitividad. La salidad de la crisis. Diaz de Santos, Madrid, 1989.

Deming, W.E.

1986 Out of the Crisis, Cambridge, Mass.: Massachusetts Institute for Technology, Center for Advanced Engineering Study.

Easton, G. y Jarrell, S.

1998 "The Effects of Total Quality Management on Corporate Performance: An Empirical Investigation”. Journal of Business, 71 (2): 253-307.

EFQM

1999 EFQM Model for Business Excellence: Company Guidelines. European Foundation for Quality Management, Bruselas.

Feigenbaum, A.V

1991 Total Quality Control. 3rd edition, megraw-Hill, New York, NY.

Flynn, B.B., Schroeder, R.C. y Sakakibara, S.

1994 "A framework for quality management research and an associated measurement instrument". Journal of Operations Management, 11: 339-366.

Forza, C. y Flippini, R.

1998 "TQM impact on quality conformance and customer satisfaction: a causal model". International Journal of Production Economics, 55 (1): 1-20.

Grande, I. y Abascal, E.

1999 Fundamentos y Técnicas de Investigación Comercial, $4^{\circ}$ edición, ESIC, Madrid.

Grandzol, J.R. y Gershon, M.

1998 "A survey instrument for standardizing TQM modeling resarch". International Journal of Quality Science, 3 (1): 80-105.

Hair, J., Anderson, R., Tathaman, R. y Black, W.

1999 Análisis Multivariante. $5^{\mathrm{a}}$ edicción, Prentice Hall, Madrid.

Ishikawa, $\mathrm{K}$.

1976 Guide to Quality Control. Asian Productivity Organization/UNIPUB, Nueva York. La primera edición japonesa data de 1968, siendo la de 1976 una edicion revisada de aquélla. Edición española: Guía de Control de Calidad. UNIPUB, Nueva York, 1985.

Ishikawa, K.

1985 Guía de control de la calidad. UNIPUB. Madrid.

Joseph, I.N., Rajendran, C., y Kamalanabhan, T.J.

1999 "An instrument for measuring total quality management implementation in manufacturing-based business units in India". Internal Journal of Production Research, 37: 2201-2215. 
Juran, J.M.

1988 Juran on Planning for Quality. The Free Press, Nueva York. Edición española: Juran y la planificacion para la Calidad. Ediciones Díaz de Santos, Madrid, 1990.

Juran, J.M. y Gyrna, F.M.

1993 Manual de control de la calidad. Madrid: mcgrawHill.

Kanji, G.K.

1998a "An innovative approach to make ISO 9000 standards more effective". Total Quality Management, 9 (1): $67-78$.

Kanji, G.K

1998b "Measurement of Business Excellence". Total Quality Management, 9 (7): 633-643.

Leal, A.

1997 "Gestión de Calidad Total en Empresas Españolas: Un análisis Cultural y de Rendimiento". Revista Europea de Dirección y Economía de la Empresa, 6 (1):. 37-56.

$\mathrm{Li}, \mathrm{L}$.

1997 "Relationships between determinants of hospital quality management and service quality performance: A path analytic model". Omega, 25: 535-545.

Luque, T.

2000 Técnicas de Análisis de Datos e Investigación de Mercados. Ediciones Piramide, Madrid.

Motwani, J

2001 "Critical factors and performance measures of total quality management”. The TQM Magazine, 13 (4): 292-300.

Nunnally, J.

1978 Psychometric Theory, mcgraw-Hill, New York.

Nurosis, M.J.

1993 SPSS. Statistical Data Análisis. SPSS Inc.

Powell, T.C.

1995 "Total quality management as competitive advantage: A review and empirical study". Strategic Management Journal, 16 (1): 15-37.

Quazi, H.A. y Padibjo, S.R.

1998 "A journey toward total quality management through ISO 9000 certification - a study on small- and medium-sized enterprises in Singapore". International Journal of Quality \& Reliability Management, 15 (5): 489-508.

Rao, S.S., Solis, L.E. y Raghunathan, T.S.

1999 "A Framework for International Quality Management Research: Development and Validation of a Measurement Instrument". Total Quality Management, 10 (7): 1047-1075.

Ritchie, L. y Dale, B.G.

2000 "Self-assessment using the business excellence model: A study of practice and process". International Journal of Production Economics, 66 (3): 241-254

Sánchez Pérez, M. y Sarabia Sánchez, F.J.

1999 "Validez y fiabilidad de escalas". En Sarabia, F.J. (coord..), Metodología para la Investigación en Marketing y Administración de Empresas. Ed. Pirámide, Madrid.

Santos, L. y Escanciano, C.

2002 "Benefits of the ISO 9000:1994 system: Some considerations to reinforce competitive advantage". International Journal of Quality \& Reliability Manage- ment, 19 (3): 321-344.

Saraph, J.V., Benson, P.G. y Schroeder, R.G.

1989 "An instrument for measuring the critical factors of quality management". Decision Sciences, .20: 810-829.

Sitkin, K., Suthleen, K. y Schroeder, R.G.

1994 "Distinguishing Control from Learning in Total Quality Management: A Contingency Perspective". Academy of Management Review, 19 (3): 537-564.

Sureshchandar, G.S., Rajendran, C. y Anantharaman, R.N.

2001, "A conceptual model for total quality management in service organizations". Total Quality Management, 12 (3): 343-363.

Tamimi, N. 1998 "A second-order factor analysis of critical TQM factors". Internarional Journal of Quality Science, 3 (1): 71-79.

Thiagarajan, T. y Zairi, M.

1998 "An empirical analysis of critical factors of TQM: A proposed tools for self-as-sessment and venchmarking pursoses". Benchmarking for Quality Management and Technology, 5: 291-303.

Wilkinson, G. y Dale, B.G.

1998 "System integration: the views and activities of certification bodies”. The TQM Magazine, 10 (4): 288-292. Zhang, Z.

2000 "Quality management in China”. The TQM Magazine, 12 (2): 92-105.

Zhang, Z., Waszink, A. y Wijngaard, J.

2000 "An instrument for measuring TQM implementation for Chinese manufacturing companies". International Journal of Quality and Reliability Management, 17: 730-755.

\section{Notas}

1 El material elaborado y puesto a disposición por la propia Fundación Europea para la Gestión de la Calidad es más que suficiente para la construcción de los ítems.

2 Este coeficiente evalúa la consistencia interna de la escala a través de la correlación media de cada una de las variables con el resto de la escala. Se basa en la consideración de que si las variables no están positivamente correlacionadas entre sí, no es probable que puedan estarlo con otras posibles variables que se pudieran seleccionar de igual manera.

3 Según Hair et al. (1999), cargas factoriales mayores a +-0,3 se consideran mínimas; las cargas de +-0,4 son importantes; las de $+-0,5$ o mayores son prácticamente significativas.

Recibido:

$21 / 01 / 2011$

Reenviado:

$10 / 01 / 2012$

Aceptado:

$13 / 01 / 2012$

Sometido a evaluación por pares anónimos 